\title{
Memory, serial anticipation pattern learning, and transfer in rats
}

\author{
E. J. CAPALDI, DONNA R. VERRY, and T. L. DAVIDSON \\ Purdue University, West Lafayette, Indiana 47907
}

\begin{abstract}
Three experiments tested hypotheses about whether rats respond appropriately to, or track, an orderly series of reward magnitudes terminating in nonreward by encoding the rule structure of the series, as a recent view derived from human serial-pattern learning models suggests, or whether they employ the memory of one reward event as a cue to signal the next reward event in the series, as animal partial-reinforcement investigations suggest. The memory signaling the terminal nonreward was called nonreward memory. The capacity of the nonreward memory to also signal reward was varied indirectly in Experiments 1 and 2, through stimulus generalization from other memories that were rewarded, and directly in Experiment 3, by rewarding it in an initial preshift phase. Consistent with a memory view, tracking of the terminal nonreward event decreased as the nonreward memory increasingly also signaled reward. These findings indicate that serial-pattern learning in rats has much in common with other, better investigated forms of instrumental learning, such as partial reward, and is not a unique form of learning, as suggested by the rule-encoding view, which was strongly disconfirmed.
\end{abstract}

How do rats manage to anticipate the terminal 0 -pellet reward in a monotonically decreasing series of reward magnitudes? Two theories, derived from very different data and having quite different implications for animal learning, have been identified as providing the basis for understanding serial-pattern learning in rats. According to Hulse (1978), human serial-pattern learning provides the basis because recent data from his laboratory demonstrate, in his view, that rats master serial patterns as people do, by encoding or inducing their rule structure (Hulse $\&$ Dorsky, 1977, 1979). According to Capaldi and Molina (1979; see also Capaldi, Blitzer, \& Molina, 1979), partial reinforcement investigations with animals provide the basis because those investigations clearly isolate the anticipatory mechanism in serial learning, and in instrumental learning generallymemory of the prior reward event. As seen by Hulse and Dorsky (1977), serial-pattern learning is a unique form of learning of considerable importance, one that has been misunderstood and ignored in animal learning. The memory view, while recognizing the importance of serial-pattern learning, sees it as continuous with other, more extensively investigated (and in that limited sense, better understood) forms of instrumental learning. The major purpose of the investigations reported here was to clearly determine,

This research was supported by NSF Grant BNS 80-01171 to E. J. Capaldi.

$A$ comment on this paper and a reply appear in the Notes and Comment section of this issue.

Editor as past investigators have not, whether the variables regulating serial-pattern learning in rats are those identified by the rule encoding view or the memory view. Ambiguity exists on this score in part because the limited data supporting the respective positions were collected under somewhat different experimental conditions and, in part, because systematic hypothesistesting research is lacking.

According to Hulse (1978), all human rule encoding models suggest that a rule such as "elements of this series decrease monotonically," written as E(i) > $E(i+1)$, is simpler than, say, a nonmonotonic rule in which elements increase as well as decrease. Simplicity of rule structure is a key variable in all rule encoding models, according to Hulse and Dorsky (1977), as is congruence between old and new rule structures in transfer situations (Hulse \& Dorsky, 1979). According to Hulse (1978), two relationships, well established in human learning-that simpler rules are encoded more rapidly than more complex ones and that positive transfer increases as congruence between old and new rule structures increaseswere recently obtained in his laboratory employing rats, thereby establishing major and important functional similarities between rats and people. First, Hulse and Dorsky (1977) reported that the strongly monotonic decreasing series of $.045-\mathrm{g}$ food pellets, 14-7-3-1-0, more readily produced slow running to the 0-pellet "reward" (tracking) than did either the nonmonotonic series, 14-1-3-7-0 (Experiment 1), or the mildly nonmonotonic series, 14-5-5-1-0 (Experiment 2). In another investigation, Hulse and Dorsky (1979) provided either monotonic or random series 
in original learning and reported that in transfer 0 pellets were better tracked in a new monotonic series when previous training was monotonic and better tracked in a new nonmonotonic series when previous training was random. According to Hulse and Dorsky (1979), these findings indicate that, in serial-pattern learning, transfer will be positive when old and new rule structures are congruent and negative when they are not.

The memory view suggests that in the series 14-7$3-1-0, S^{14}$, the memory of 14-pellet reward, is retrieved on 7-pellet trials and so becomes a cue, or signal, for anticipating a 7-pellet reward. Similarly, $S^{7}$ signals 3 pellets, $S^{3}, 1$ pellet, and $S^{1}, 0$ pellets. Tracking of 0 pellets in the 14-7-3-1-0 series ultimately depends, then, upon $S^{1}$ being retrieved on 0-pellet trials. But extinction studies suggest that, in a series such as 14-7-3-1-0, $S^{1}$ will come to signal not only nonreward but reward as well, and the stronger the capacity of $S^{1}$ to signal reward, the more vigorously will the animal respond to the 0 -pellet event, that is, the poorer will be tracking. According to such investigations, increases in the capacity of a memory to signal reward occur, as with other cues, in one of two general ways: directly, by being retrieved on rewarded trials, or indirectly, through stimulus generalization from other memories that were retrieved on rewarded trials. Considering direct increases, the capacity of a memory to signal reward increases the more often it is retrieved on rewarded trials (Capaldi, 1964) and the greater the magnitude of reward on the rewarded trials (e.g., Capaldi \& Capaldi, 1970; Leonard, 1969). The generalized reward-signal capacity accruing to a memory will increase, as is the case for other cues, as the strength or capacity of generalized stimuli to signal reward increases, as the similarity of the generalized stimuli increases, and as the number of generalized stimuli increases, since several generalized tendencies can summate (see Capaldi, 1964, 1966). By way of example, in the series 14-7-3-1-0, $\mathrm{S}^{1}$, the memory of nonreward (hereinafter referred to as "nonrewarded memory") would receive generalized reward signal strength from each of three "rewarded memories," $S^{14}, S^{7}$, and $S^{3}, S^{1}$ being most similar to $S^{3}$ and least similar to $S^{14}$. And $S^{14}$ would directly acquire the greatest reward signal strength, since, among the reward memories, it signals the largest reward, 7 pellets.

As defined by Hulse and Dorsky (1977), in presenting the 14-7-3-1-0 series, we have one trial consisting of five runs. That terminology is henceforth employed here to facilitate comparison with their investigation. Hulse and Dorsky $(1977,1979)$ presented several trials each day, the trials being separated by a longer interval $(10-15 \mathrm{~min})$ than were the runs $(10-15 \mathrm{sec})$. These procedures were followed here.

\section{EXPERIMENT 1}

Consider the case in which some memory, $\mathrm{X}$, sig- nals nonreward and another, similar memory, $Y$, signals reward. According to a generalization analysis, the nonrewarded memory, $\mathrm{X}$, will receive a generalized capacity to signal reward from the similar rewarded memory, $Y$, which will reduce tracking of 0 pellets. This implication of a generalization analysis was confirmed by Capaldi et al.(1979). That investigation compared several two- and three-element series, an example of each being the series 4-0 and 16-4-0, respectively. In both series, $\mathrm{S}^{4}$ signals nonreward. However, in the 16-4-0 series, $S^{16}$ signals 4 pellets and, by supplying some of this reward-signal capacity to $S^{4}$ through stimulus generalization, should reduce tracking. These were the results obtained by Capaldi et al.(1979), the two-element series producing better tracking of 0 pellets than the three-element series.

On the reasonable assumption that an increased number of instances of a rule should facilitate its encoding, the findings of Capaldi et al. (1979) may be seen as incompatible with the rule encoding view. The 16-4-0 series contains two instances of the rule $E(i)>E(i+1)$, the $16-4$ transition and the 4-0 transition, while the 4-0 sequence contains, of course, only one instance of that rule. The failure of the additional 16-4 sequence to promote better tracking of 0 pellets, while consistent with a generalization approach, may not necessarily disconfirm the rule encoding view. Capaldi et al. (1979) suggested that it might be more difficult to "locate" or determine when the terminal 0-pellet element is to occur in longer three-element series than in shorter two-element series. According to this view, as series length increases, an increase in problem difficulty may occur, uncompensated for by the increase in number of rule instances. Experiment 1 attempted to provide a clearer test of the memory-generalization and rule-encoding views by pitting stimulus generalization against number of rule instances while equating the difficulty of location factor. This was accomplished as follows. Group 4-0 received the single trial each day, 4-0. Group 16-4/4-0 received two trials each day, a 16-4 trial and a 4-0 trial, these trials being separated by a 30-min interval. Concern, of course, was with tracking on the common 4-0 trial. Thus, the major question in Experiment 1 was: Would the additional 16-4 trial facilitate tracking on the 4-0 trial, as a ruleencoding analysis appears to suggest, or hinder it, as a stimulus-generalization analysis suggests? Two additional groups were employed in Experiment 1; one received the series 16-4-0 each day and the other received the series 16-4.

\section{Method}

Subjects. The subjects were 32 naive male Holtzman rats, nurchased from the Holtzman Co., Madison, Wisconsin, that were about 100 days old at the start of the experiment.

Apparatus. The apparatus was a runway, $202.3 \mathrm{~cm} 10 n g$, $11.1 \mathrm{~cm}$ wide, and $22.9 \mathrm{~cm}$ high, painted flat gray. The initial $19.1 \mathrm{~cm}$ of the runway, separated by a guillotine-type door, served 
as a startbox. Operation of the start door initiated the first of three clocks $(.01 \mathrm{sec})$. Clock 1 was stopped (start time) and clock 2 was started when the rat interrupted a photobeam $6.35 \mathrm{~cm}$ from the start door. Clock 2 was stopped (run time) and clock 3 was started when the rat interrupted a second photobeam $132.1 \mathrm{~cm}$ from the first. Clock 3 was stopped (goal time) when the rat interrupted a third photobeam $33.4 \mathrm{~cm}$ from the second. The last $29.8 \mathrm{~cm}$ of the runway served as a goalbox in which the rat could be confined by the lowering of a guillotine-type door. The goalbox contained a brass cup that was removable. The entire apparatus was covered by wire mesh on a hinged frame.

Pretraining. Upon arrival in the laboratory, the subjects were housed individually and given free access to Lab Blox and water for 10 days. Pretraining began 12 days prior to experimental training. On Day 1 of pretraining, the $13 \mathrm{~g}$ /day deprivation schedule, which was maintained throughout the experiment, was begun. On each day of pretraining, each rat was removed from the home cage for about $2 \mathrm{~min}$ and handled. On Days 10, 11, and 12, each rat received $16.045 \mathrm{~g}$ Noyes pellets in the home cage. On Days 11 and 12 of pretraining, each rat was allowed to explore the unbaited alley for a few minutes. The number of pellets received by the rat in pretraining, as in experimental training, was subtracted from the 13-g daily ration.

Fxperimental training. The rats were divided ino four groups of eight each. Three of the groups, 4-0, 16-4, and 16-4-0, received one trial each day. The groun designations describe the reward Ireatment: Group 4-0, for example, received 4 pellets on its first run and 0 pellets on its second run. The fourth group, 16-4/4-0, received two trials each day, a 4-0 trial initially and, about $30 \mathrm{~min}$ later, a $16-4$ trial. This procedure, as will become clear below, insured that exactly the same experimental conditions would prevail on 4-0 trials for Groups 16-4/4-0 and Group 4-0. The comparison of these groups on 4-0 trials was, of course, the major purpose of Experiment 1.

The rats were taken into the experimental room in squads of eight, two rats from each group. There were four different orders of runs within a squad, with each squad being run at the same time each day. A rat was given all of the runs of its scheduled trial before the next rat was run, the interval between runs being about $15-20 \mathrm{sec}$. In order to insure a minimum 30 -min interval between trials, after all the rats in group 16-4/4-0 were run, four rats in an entirely unrelated experiment were run. After the rats from the unrelated experiment were run, the rats in Group 16-4/4-0 were given their $16-4$ trial. Thus, the $4-0$ trial was received by Groups $16-4 / 4-0$ and 4-0 under exactly the same experimental conditions.

The rats were removed from the goalbox immediately after they had eaten the pellets or were confined to the goalbox for $20 \mathrm{sec}$ on nonrewarded trials. The maximum time allowed the rat to traverse each alley section was $60 \mathrm{sec}$. If the rat failed to traverse the runway in the alloted time, it was gently placed in the goalbox to receive the scheduled reward. After the last rat of the squad was run, the squad was returned to the colony room and each rat was returned to its home cage, where, about 20 min later, it was fed the daily ration.

\section{Results}

In none of the three experiments reported here were the results obtained influenced by alley section, and, therefore, times along the total lengths of the alley are reported. Tracking may be assessed by two methods: between groups, slower running by one group than another on 0-pellet runs; and within-groups, slower running by a particular group on its 0-pellet runs than on its rewarded runs. Both methods of assessing tracking are employed here.

Tracking of 0 pellets was clearly better accomplished by Group 4-0 than by either Group 16-4/4-0 or Group 16-4-0, as is evident in Figure 1, which shows mean log running times in these groups on 4-0 trials in blocks of 2 days. Even over the first half of training, Blocks 1-4, Group 4-0 showed some tendency to track 0 pellets more effectively than did the remaining groups. By the second half of training, Blocks 5-8, Group 4-0 was clearly superior to Groups $16-4 / 4-0$ and $16-4-0$, running about as rapidly as those groups on 4-pellet runs and much more slowly on 0 -pellet runs. Statistics demonstrate the superiority of Group 4-0. Importantly, the interaction of Runs by Groups was significant at the .05 level or better on each of Blocks 3-8 [smallest $\mathrm{F}(1,21)=4.19$ on Block 3]. The basis of this interaction, as revealed by subsequent Newman-Keuls tests, is less clear on Blocks 3 and 4 than on the latter blocks, because on these early blocks Group 4-0 was significantly slower than the remaining groups on 4-pellet as well as on 0-pellet runs (ps $<.05$ ). However, on Blocks 5-8 there was absolutely no question that the basis of the Runs by Groups interaction was better tracking in Group 4-0 than in the remaining groups, since on each of these blocks the groups did not differ significantly on 4pellet runs but did differ on 0-pellet runs, Group 4-0, of course, running more slowly on 0 -pellet runs than either Group 16-4-0 or Group 16-4/4-0 (ps < .05 or better). Of some interest, too, are within-groups differences, tracking as revealed by slower running on 0-pellet than on 4-pellet runs in each group. At one extreme, Group 4-0 showed slower running on 0-pellet than on 4-pellet runs on each of Blocks 5-8 (ps $<.01$ ). At the other extreme, Group 16-4-0 failed to track on any block. Group 16-4/4-0 was intermediate, running more slowly on 0-pellet than on 4-pellet runs on Blocks 5 and 8 (ps $<.05$ ). Thus, on both a between-groups and a within-groups basis, Group 4-0 tracked 0 pellets better than either of the remaining groups.

Groups 16-4/4-0, 16-4-0, and 16-4 showed little tendency to differ on 16-4 trials, as is evident in Figure 2 , which shows mean log running time on 16-4

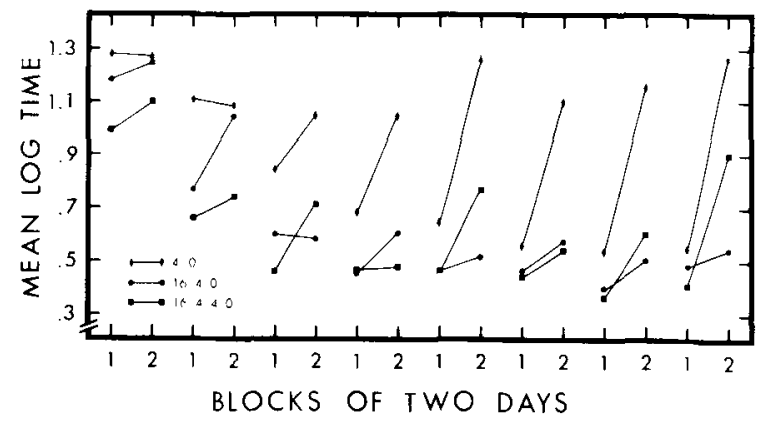

Figure 1. Mean log running times in the alley in blocks of 2 days in the three groups that received 0-pellet Irials. Groups 4-0, 16-4-n, and 16-4/4-0. For each of these groups on each of the 2-day hlocks, running times are shown on 4-pellet runs (the first point plotled in each block) and on 0-pellet runs (the second point plotted in each block). 


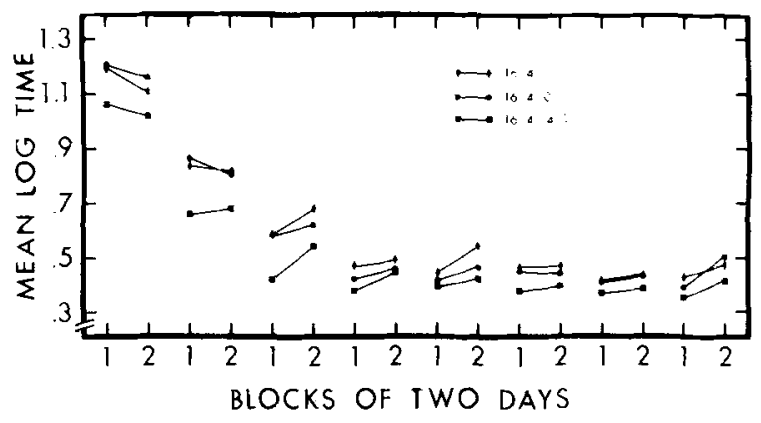

Figure 2. Mean log running times in the alley in blocks of 2 days in the three groups that received the 16-4 sequence, Groups 16-4, 16-4-0, and 16-4/4-0. For each of these groups on each of the 2-day blocks, running times are shown on 16-pellet runs (the first point plotted in each block) and on 4-pellet runs (the second point plotted in each block).

trials in these groups in blocks of 2 days. The tendency in those groups was to speed up over blocks, but on each block to run as rapidly on the 4-pellet as on the 16-pellet runs. On none of the trial blocks were differences between Groups 16-4/4-0, 16-4-0, and 16-4 significant at the .05 level or better on $16-4$ trials.

\section{Discussion}

Necessarily, according to a rule-encoding view, the greater the number of instances of a rule, the faster it should be encoded. According to a memory analysis, however, increased instances of a rule may be counterproductive with respect to tracking, if they result in increased generalization of reward signal capacity to the nonrewarded memory. In Experiment 1, Group 16-4/4-0 received two instances daily of the rule $E(i)>E(i+1)$, while Group 4-0 received only one daily instance of the rule. However, in Group 16-4/ $4-0, S^{16}$, which may be conceptualized as an $\mathrm{S}+$ cue, since it was retrieved on rewarded trials, supplied $\mathrm{S}^{4}$, which may be conceptualized as an $\mathrm{S}$ - cue, since it was retrieved on nonrewarded trials, with generalized reward signal capacity, while in Group 4-0 there was not, so to speak, any $\mathrm{S}+$ cue to supply $\mathrm{S}^{4}$ with generalized reward signal capacity. It was found, in Experiment 1, that 0 pellets was better tracked by Group 4-0 than by Group 16-4/4-0. Thus, the results of Experiment 1 confirm a memory-generalization view and appear to disconfirm a rule-encoding view. That $\mathrm{S}^{16}$ did, indeed, possess considerable reward signal capacity is suggested by the internal evidence available in Experiment 1. Group 16-4/4-0, when given its 16-4 trial, ran rapidly on 4-pellet runs. Also, Groups 16-4 and 16-4-0 ran rapidly on 4-pellet runs as well. Better tracking of 0 pellets by Group 4-0 than by Group 16-4-0 confirms an earlier finding of Capaldi et al. (1979).

A rule approach might be able to deal with the results obtained in Experiment $\mathbf{l}$ by suggesting that groups that receive two daily trials, such as Group
16-4/4-0, have a more difficult problem than do groups that receive one daily trial, such as Group 4-0. Consider two of perhaps numerous possible reasons why this might be so. In Group 4-0, 0 pellets may be tracked not only by employing the rule $E(i)>$ $E(i+1)$, but also by employing the rule that the second run of the day is always nonrewarded. In Group 16-4/4-0, the former rule could be employed, but not the latter. Thus Group 4-0 may have tracked 0 pellets better than Group 16-4/4-0 because the rule that the second run of the day is nonrewarded is simpler than the rule $E(i)>E(i+1)$. Or both of these rules may have been encoded by Group 4-0, making tracking of 0 pellets simpler on that basis. Experiment 2 examined the rule-encoding and generalization approaches when all groups received two trials each day.

\section{EXPERIMENT 2}

From a stimulus generalization point of view, Experiment 2 is a simple extension of Experiment 1 . While Experiment 1 examined the effects of the presence or absence of a rewarded Y memory on a nonrewarded $\mathrm{X}$ memory, in Experiment 2 all groups received the $X$ and $Y$ memories with their similarities being varied. The four groups of Experiment 2 were trained as was Group 16-4/4-0 of Experiment 1, receiving two two-run trials daily, one terminating in reward and the other in nonreward. Call a trial such as the 16-4 trial of Group 16-4/4-0 a sequence that terminates in reward, or, more simply, a rewarded sequence. Correspondingly, we may refer to a trial such as the 4-0 trial as a nonrewarded sequence.

In Experiment 2, the two-element nonrewarded sequence necessarily conformed to the rule $\mathrm{E}(\mathrm{i})>$ $\mathrm{E}(\mathrm{i}+1)$. However, while the rewarded sequence conformed to this rule in two of the groups, it conformed to the opposite rule, $\mathrm{E}(\mathrm{i})<\mathrm{E}(\mathrm{i}+1)$, in the remaining two groups. The rule-encoding view predicts faster tracking of 0 pellets when the two daily sequences conform to the same rule, because when rule instances are contradictory two incompatible rules must be encoded. According to stimulus generalization, however, consistency of rule instances is irrelevant to tracking. What matters is how much reward-signal capacity generalizes from the rewarded to the nonrewarded memory. In Experiment 2, such generalization was determined by similarity between the rewarded and nonrewarded memories. Experiment 2 attempted to determine whether tracking is determined by consistency of rule instance or by the capacity of the nonrewarded memory to signal reward as determined by stimulus generalization.

\section{Method}

Subjects. The subjects were 40 rats as described in Experiment 1

Apparatus. The apparatus was the same as that used in F.xperiment 1. 
Pretraining. Pretraining was as in Experiment 1.

Experimental training. The rats were divided into four groups of 10 rats each. All groups received two trials each day, each trial consisting of two runs. The four groups were designated as $1-10 / 2-0,1-10 / 20-0,15-10 / 2-0$, and $15-10 / 20-0$. The group designations describe treatment. Group 1-10/2-0, for example, received daily a 1-10 trial (a rewarded sequence) and a 2-0 trial (a nonrewarded sequence). For each group, the rewarded sequence was initially given twice (Days 1 and 4 of each 4-day block) and subsequent to the nonrewarded sequence twice in each block. The rats were taken into the experimental room in squads of eight, two from each group. The first rat in a squad was given its trial before the second rat was given its trial, and so on. After the last rat in a squad had been given its first trial, the first rat in the squad was given its second trial, and so on. This produced an interval of about 15-20 min between trials. The order in which each of the five squads was run each day was fixed, but the order of trials within a squad was randomized daily. All other aspects of experimental training were as in Experiment 1.

\section{Results}

The results obtained in Experiment 2 were entirely clear. Groups 1-10/20-0 and 15-10/2-0 tracked 0 pellets and differed from each other only minimally; Groups $15-10 / 20-0$ and 1-10/2-0 failed to track 0 pellets and differed from each other only minimally, as indicated in Figure 3, which presents mean log running times on the nonrewarded sequence in blocks of 4 days. Over the first half of training, Blocks 1-3, the major tendency in all groups was to speed up over blocks. However, in the second half of training, Blocks 4-6, tracking of 0 pellets emerged strongly in Groups 1-10/20-0 and 15-10/2-0 and failed to emerge in Groups $15-10 / 20-0$ and 1-10/2-0. On each of Blocks 4-6, the interaction of the nonrewarded trial sequence and the rewarded trial sequence was significant at the .01 level or better $[F s(1,36)=11.28,13.01$, and 22.96 on Blocks 4, 5, and 6, respectively]. NewmanKeuls tests indicated that on none of the blocks did the groups differ on the rewarded run of the sequence. However, on 0-pellet runs on Blocks 5 and 6, Groups

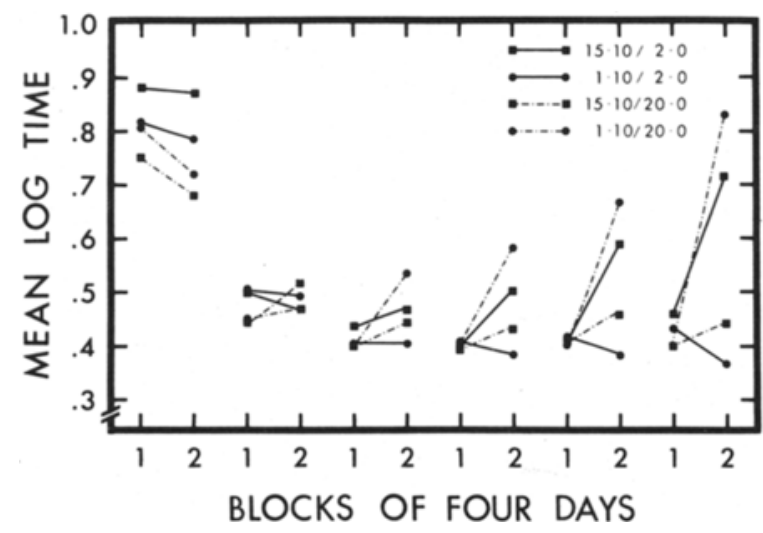

Figure 3. Mean log running time in the alley in blocks of 4 days in each of the groups on the rewarded run and on the nonrewarded run of the nonrewarded sequence. The rewarded run is the first point plotted in each block, the nonrewarded run is the second point.

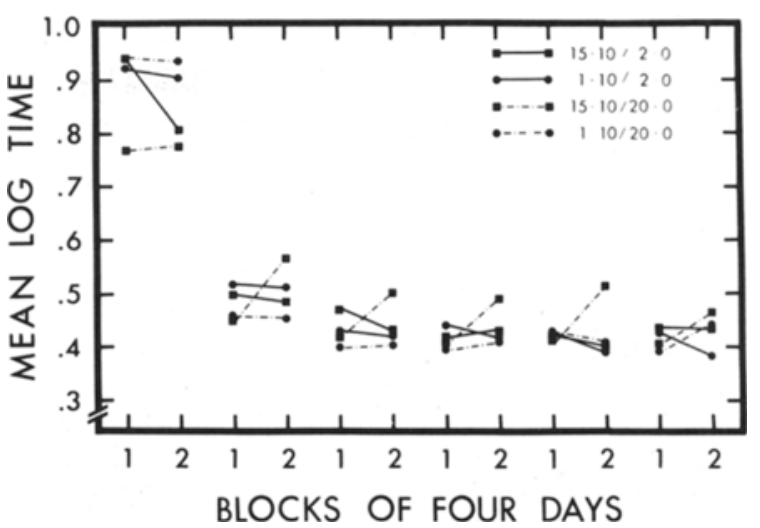

Figure 4. Mean log nuning time in the alley in blocks of 4 days in each of the groups on the two runs of the rewarded sequence. The 1- or the 15-pellet run is the first point plotted in each block, and the 10-pellet run is the second point.

1-10/20-0 and 15-10/2-0, which did not differ from each other, ran more slowly than Groups 15-10/20-0 and 1-10/2-0 (ps $<.01$ ), which also did not differ from each other. Tracking on a within-groups basis, slower running by a particular group on its nonrewarded than on its rewarded run of the sequence was shown by Groups $1-10 / 20-0$ and $15-10 / 2-0$ on Blocks 4, 5, and 6 (ps <.05). However, on none of the trial blocks was such tracking shown by either Group 15-10/20-0 or Group 1-10/2-0. Thus, these latter groups showed no tendency to track 0 pellets by the termination of the experiment.

Running on the rewarded sequence in blocks of 4 days is shown in Figure 4. The groups differed little on the rewarded sequence. There was some tendency in the middle trial blocks for Group 15-10/20-0 to slow up on the 10-pellet runs, but this effect was too small to be noted statistically, and on none of the trial blocks was there a significant effect due either to groups or to the interaction of the rewarded and nonrewarded sequence.

\section{Discussion}

In Experiment 2, consistency of rule instances was irrelevant to tracking, but stimulus generalization from the rewarded to the nonrewarded memory, determined by their similarity, was not. In Groups $15-10 / 20-0$ and $15-10 / 2-0$, both daily trials were consistent with the rule $\mathrm{E}(\mathrm{i})>\mathrm{E}(\mathrm{i}+1)$. But, in Groups $1-10 / 20-0$ and $1-10 / 2-0$, while one daily trial was consistent with the rule $\mathrm{E}(\mathrm{i})>\mathrm{E}(\mathrm{i}+1)$, the other daily trial was consistent with the opposite rule, $\mathrm{E}(\mathrm{i})<\mathrm{E}(\mathrm{i}+1)$. According to the rules approach, simpler rule structures, such as "elements always decrease monotonically," should be encoded more rapidly than more complex rule structures, such as "elements sometimes decrease and sometimes increase" (Hulse, 1978; Hulse \& Dorsky, 1977, 1979). Thus, according to the rules approach, 0 pellets should 
have been better tracked in Groups 15-10/20-0 and $15-10 / 2-0$ than in Groups 1-10/20-0 and 1-10/2-0. While 0 pellets was better tracked by Group $15-10 / 2-0$ than by Group 1-10/2-0, better tracking of 0 pellets by Group 1-10/20-0 than by Group 15-10/20-0 demonstrates that, in Experiment 2, consistency of rule instances was irrelevant to tracking.

The results obtained in Experiment 2 are completely consistent with a generalization analysis and may be understood as follows: The more similar a reward memory is to a nonreward memory, the greater the reward-signal capacity supplied to the nonreward memory, and thus the poorer should be the tracking of 0 pellets. Consider first Groups 15-10/ 2-0 and 1-10/2-0. In Group 15-10/2-0, $\mathrm{S}^{15}$ signaled 10 pellets and $S^{2}, 0$ pellets, while in Group $1-10 / 2-0$, $S^{1}$ signaled 10 pellets and $S^{2}, 0$ pellets. Since $S^{1}$ and $S^{2}$ are more similar than $S^{15}$ and $S^{2}, 0$ pellets was more poorly tracked by Group 1-10/2-0 than by Group 15-10/2-0. Poorer tracking of 0 pellets in Group 15-10/20-0 than in Group 1-10/20-0 may be understood on exactly the same basis: $S^{15}$ and $S^{20}$ are more similar than $\mathbf{S}^{\mathbf{1}}$ and $\mathrm{S}^{\mathbf{2 0}}$.

Better tracking of 0 pellets in Group 1-10/20-0 than in Group 15-10/20-0 seems to effectively disconfirm the rule-encoding hypothesis. First of all, there can be no question of 0 pellets' being easier to "locate" in one series than in the other, because both groups received the identical 20-0 series. Second, according to rule encoding, Group 1-10/20-0, for which elements both increased and decreased, had a harder, not an easier, problem than Group 15-10/ 20-0, for which elements always decreased. What was common to Experiments 1 and 2, then, is that in both of them tracking of 0 pellets decreased along with an increase in the generalized reward-signal capacity supplied to the nonreward memory. On this basis, it is reasonable to conclude that Experiment 1 demonstrated that number of rule instances is irrelevant to tracking of 0 pellets and that Experiment 2 demonstrated that consistency of rule instances is irrelevant as well.

An adequate test of the rule-encoding view and the memory view was made possible by emphasizing the comparisons above, that between the two groups in which $S^{2}$ signaled 0 pellets, Groups $1-10 / 2-0$ and $15-10 / 2-0$, and that between the two groups in which $\mathrm{S}^{20}$ signaled 0 pellets, Groups 1-10/20-0 and 15-10/ 20-0. However, caution is urged in comparing groups in which $S^{2}$ signaled 0 pellets with groups in which $S^{20}$ signaled 0 pellets, because those groups differed along two dimensions, the memory signaling 0 pellets and the amount of generalization from the rewarded to the nonrewarded memory. Consider, for example, Groups 1-10/20-0 and 1-10/2-0. Different memories signaled 0 pellets in the two groups, either $\mathrm{S}^{20}$ or $\mathrm{S}^{2}$, and there is reason to believe that this variable might affect tracking, although what this in- fluence might be is unclear (see, e.g., Capaldi, Berg, $\&$ Morris, 1975). Moreover, less generalization of reward signal strength occurred in Group 1-10/20-0 (from $S^{1}$ to $S^{20}$ ) than in Group 1-10/2-0 (from $S^{1}$ to $S^{2}$ ). In any event, it should be mentioned that the memory view is in no way compromised when groups in which $\mathrm{S}^{2}$ signaled 0 pellets are compared with groups in which $\mathrm{S}^{20}$ signaled 0 pellets, a matter which is perhaps clear.

\section{EXPERIMENT 3}

The memory view of extinction (e.g., Capaldi, 1966), when modified in a relatively minor way, may be able to explain transfer in serial-learning tasks. In extinction, only the memory of nonreward occurs and it signals nonreward, while in serial-learning tasks, any memory may signal terminal nonreward, including memories produced by reward. As was clear in Experiments 1 and 2, as the capacity of a memory to signal reward increased, strength of responding increased. According to the memory view, resistance to extinction should increase, the more strongly the memory of nonreward has been made a signal for reward in acquisition, because the more strongly the memory of nonreward signals reward, the more difficult will it be to convert it into a signal for nonreward in the extinction phase. A similar difficulty may be encountered in attempting to convert memories produced by reward from signals for reward to signals for nonreward, and in serialpatterning studies memories produced by reward may be used to signal nonreward. Accordingly, if extinction studies are any guide to transfer in serial learning, and Experiments 1 and 2 suggest they are, then it may be that in transfer studies the more strongly some memory, either of reward or nonreward, has come to signal reward in original training, either because of direct association with reward or generalization from other rewarded memories, the more difficult it will be for that memory to become a signal for nonreward in transfer. Experiment 3 addressed directly the question of whether transfer in serial learning is determined by the capacity of memories to signal reward or, as Hulse and Dorsky (1979) suggest, the degree of congruence between old and new rule structures. The variables manipulated in Experiment 3 , as will be shown later, are analogous to variables that have been shown to affect resistance to extinction in partial reinforcement investigations, and it was of some interest to determine if those variables would affect transfer in a manner analogous to extinction.

\section{Method}

Subjects. The subjects were rats as described in Experiment 1. Apparatus. The apparatus was the same as that in Experiment 1. Pretraining. Pretraining was as in Experiment 1 
Experimental training-Phase 1. Half the rats were trained according to the increasing rule, $E(i)<E(i+1)$, and half according to the equality rule, $\mathrm{E}(\mathrm{i})=\mathrm{E}(\mathrm{i}+1)$. All rats received two twoelement trials daily in Phase 1 for 12 days and the same number of 2 as of 20 pellet runs. In the increasing condition, the rats received the sequence 2-20 twice each day. In the equality condition, there was one 2-2 sequence each day and one 20-20 sequence each day. Within each 4-day block, the 2-2 trial was given initially twice (Days 1 and 4 of each block) and subsequent to the 20-20 trial twice.

Experimental training-Phase 2. In Phase 2, which lasted 4 days, there were two two-element trials daily, the first run of each trial being rewarded and the second run being nonrewarded; thus, in Phase 2, the decreasing rule, $E(i)>E(i+1)$, prevailed for all rats. Half the rats given increasing training in Phase 1 received the sequence $20-0$ in Phase 2 (Group I-20), and the remaining half received the sequence 2-0 (Group I-2). Half the rats given equality training in Phase 1 received the sequence $20-0$ in Phase 2 (Group $\mathrm{E}-20$ ), and the remaining half the sequence $2-0$ (Group E-2).

In both Phase 1 and Phase 2, the rats were brought into the experimental room in squads of four, one from each group. Each rat was given its trial before the next rat in the squad was run, the order of runs within a squad being randomized daily. There were two experimenters, each of whom ran four squads. When the last rat in the first squad had been given its first trial of the day, the squad was returned to the colony room, and the same procedure was repeated with the second, third, and fourth squads, the order of runs for the four squads each day being fixed. After Squad 4 had been given its first trial, Squad 1 was given its second trial, followed, in order, by Squads 2, 3, and 4. All other aspects of the procedure were as in Experiment 1.

\section{Results}

Two comparisons of major interest in Experiment 3 were that between the two groups in which $\mathrm{S}^{2}$ signaled 0 pellets in transfer, Groups E-2 and I-2, and that between the two groups in which $\mathrm{S}^{20}$ signaled 0 pellets in transfer, Groups E-20 and I-20. As indicated earlier, comparisons involving groups in which $\mathrm{S}^{2}$ signaled 0 pellets with groups in which $\mathrm{S}^{20}$ signaled 0 pellets should be approached with caution. In original training in the equality condition, per- formance did not differ on 2-2 and 20-20 trials, and in both the equality condition and the increasing condition, performance was not affected by the dummy transfer variable, so it was possible to collapse over these variables. Mean log running time in original training in the equality condition and in the increasing condition in blocks of 2 days appears in the left panel of Figure 5; performance in transfer in each of the four groups on each of the 5 days of transfer is shown in the right panel of Figure 5. By the end of original training, as Figure 5 shows, performance reflected reward conditions, rats in the equality condition running equally rapidly on both runs of their trials and rats in the increasing condition showing a decrease in running time over the two runs of their trials. Figure 5 also shows that, later in training, rats in the increasing condition ran more slowly than rats in the equality condition on the first run of the sequence, while they were practically identical in the two conditions on the second run of the sequence. Turning to statistics, on the last block of original training trials, the Runs by Groups interaction was significant $[F(1,28)=18.63, p<.001]$. Subsequent NewmanKeuls tests revealed a significant decrease in running time over runs in the increasing condition $(p<.01)$ but not in the equality condition and a significant difference between the groups on the first run of the sequence $(p<.01)$ but not the second.

It is important to note that the groups did not differ on the second run of the sequence either at the end of Phase 1 or, as can be seen in Figure 5, on the first day of transfer; thus, such difference as occured on 0-pellet runs in transfer developed during the course of transfer training. The tracking of 0 pellets in transfer was better in Group E-2 than in Group I-2, and it was better in Group I-20 than in Group E-20. The

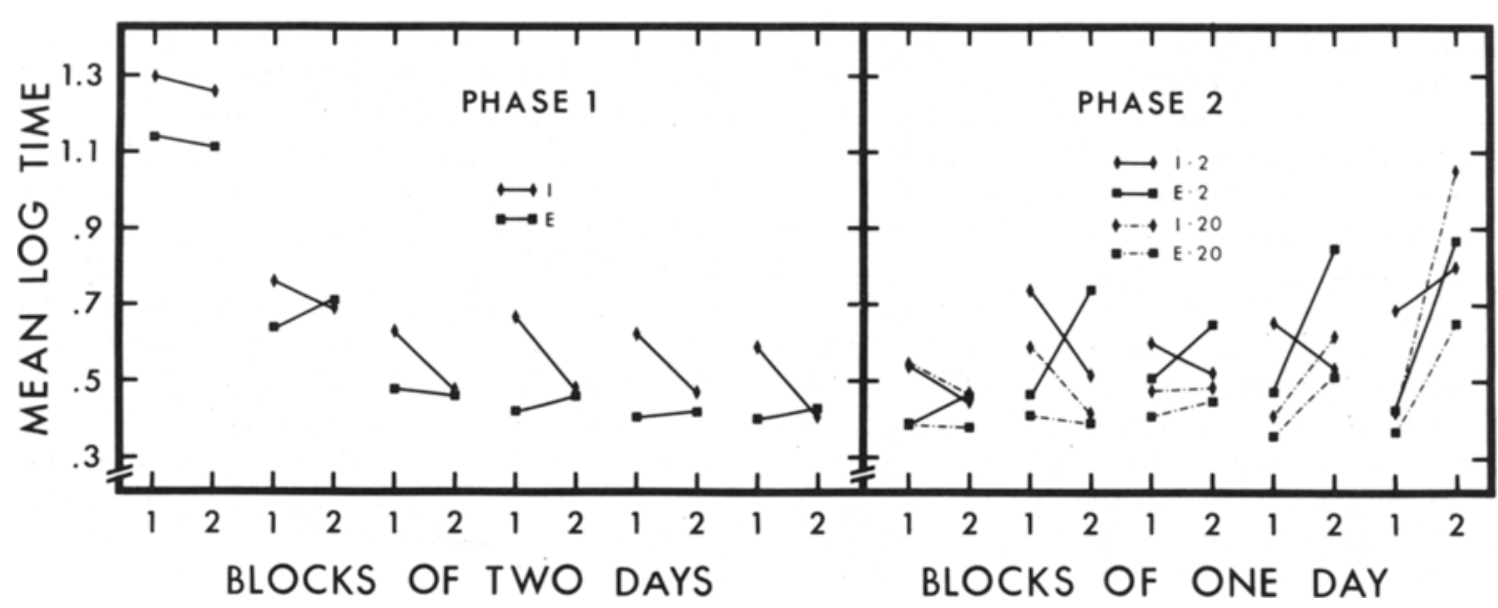

Figure 5. The left panel shows mean log running time in the alley in blocks of 2 days in the equality condition (E) and in the increasing condition (I), the first point plotted in each block being the first run of the sequence and the second point being the second run of the sequence. The right panel shows mean log running time in the alley on each of the days of transfer in each of the four groups, E-2, I-2, E-20, and I-20. The rewarded run is the first point plotted in each block and the 0-pellet run is the second point plotted. 
tendency for Group E-2 to run more slowly on 0-pellet runs than Group 1-2 appeared early in training and was much reduced by the last day of transfer. However, the opposite was the case for Groups E-20 and $\mathrm{I}-20$, in which the tendency for these groups to differ on 0-pellet runs was largest on the last day of transfer.

The Runs by Groups interaction was significant on each of the 5 days of transfer [smallest $F(1,28)=$ $4.53, \mathrm{p}<.05$, on Day 3 of transfer]. Subsequent Newman-Keuls tests clearly demonstrate that the tracking of 0 pellets was better in Group E-2 than in Group I-2 and better in Group I-20 than in Group E-20. Consider Groups E-2 and 1-2 first. On 0-pellet runs, Group E-2 was slower than Group I-2 on Days 2,3 , and 4 of transfer ( $p s<.05$ or better). If anything, within-group comparisons even more strongly demonstrate the tracking superiority of Group E-2 relative to Group 1-2. Group E-2 was slower on its 0 -pellet runs than on its 2-pellet runs on Days 2, 3, 4, and 5 of transfer (ps $<.05$ or better). However, only by the last day of transfer was Group I-2 slower on 0 -pellet than on 2-pellet runs, and that difference was not significant. Consider now Groups E-20 and I-20. On the last day of transfer, Group I-20 ran significantly more slowly than Group E-20 on 0pellet runs $(\mathrm{p}<.01)$, the two groups failing to differ on 20-pellet runs. On Day 5 of transfer, Group I-20 was slower on its 0 -pellet than on its 20 -pellet run $(\mathrm{p}<.01)$, as was Group E-20 $(\mathrm{p}<.05)$. However, on Day 4 of transfer, while Group I-20 ran more slowly on 0 -pellet than on 20-pellet runs $(\mathrm{p}<.05)$, Group E-20 did not. Thus, superior tracking by Group I-20 relative to Group E-20 is revealed statistically on both a between-group and a within-group basis.

\section{Discussion}

Transfer in Experiment 3 was (1) independent of the degree of congruence between old and new rule structures, (2) dependent upon the reward signal strength of memories, and (3) influenced by variables similar to those previously shown to affect partial reinforcement extinction. Considering Experiment 3 in relation to the rule-encoding view, two assumptions regarding rule congruence are possible: the decreasing rule in transfer $\mathrm{E}(\mathrm{i})>\mathrm{E}(\mathrm{i}+1)$ is more congruent with the equality rule that prevailed in Phase $1, E(i)=$ $E(i+1)$, or is more congruent with the increasing rule that prevailed in Phase $1, \mathrm{E}(\mathrm{i})<\mathrm{E}(\mathrm{i}+1)$. Under either assumption, the rule-encoding view is disconfirmed. If it is assumed that the decreasing rule is more congruent with the equality rule than with the increasing rule, then the rule-encoding view is disconfirmed by better tracking of 0 pellets in Group I-20 than in Group E-20. If the opposite assumption is made, then the rule-encoding view is disconfirmed by better tracking of 0 pellets in Group E-2 than in Group I-2. In Experiment 3, then, transfer could not possibly have been related to the degree of congruence between old and new rule structures.

The applicable memory principle in connection with Groups E-2 and I-2 is that a memory retrieved on a large-reward trial will acquire a stronger tendency to signal reward than will a memory retrieved on a small-reward trial. On this basis, poorer tracking of 0 pellets in transfer by Group I-2 than by Group $\mathrm{E}-2$ is consistent with the memory view outlined earlier, that the more strongly a memory has come to signal reward in original training, the more difficult it will be to convert that memory into a signal for nonreward in transfer; and the findings for Groups E-2 and I-2 may be shown to be entirely consistent with prior partial-reward extinction findings. In original training in Group I-2, 2-pellet runs were followed by 20 -pellet runs, and thus, in Group I-2, $\mathrm{S}^{2}$ came to directly signal 20 pellets. However, in original training in Group E-2, 2-pellet runs were followed by 2 -pellet runs, and thus, in Group E-2, $\mathrm{S}^{2}$ came to directly signal 2 pellets. In transfer, in both Group I-2 and Group E-2, the memory $S^{2}$ signaled nonreward. Thus, nonreward was better tracked by Group E-2 than by Group I-2 because in Group E-2, $\mathrm{S}^{2}$ had the weaker tendency to signal reward. Resistance to extinction following partial reward is less when, in acquisition, nonrewarded runs are followed by small-reward runs than when the nonrewarded runs are followed by large-rewarded runs (e.g., Capaldi \& Capaldi, 1970; Leonard, 1969). The memory interpretation of this extinction finding is exactly the same as that applied to the transfer findings for Groups E-2 and I-2, except that different memories are involved in the two cases, $S^{2}$ in the transfer case and the memory of nonreward in the extinction case.

In original training in Group E-20, 20-pellet runs were followed by 20-pellet runs, while, in Group I-20, the 20-pellet run ended the series. Resistance to extinction under partial reinforcement is greater when, in acquisition, nonrewarded runs are followed by rewarded runs than when the nonrewarded run ends the daily series (e.g., Capaldi \& Morris, 1976). As before, the memory interpretation of the extinction and transfer cases is identical, except that when the memory $\mathrm{S}^{20}$ is mentioned in the transfer case the memory of nonreward would be mentioned in the extinction case. The memory $\mathrm{S}^{20}$ signaled nonreward in transfer in Groups E-20 and I-20. The present view, then, is that in original training $S^{20}$ acquired a stronger tendency to signal reward in Group E-20, in which it was retrieved on 20-pellet runs, than in Group 1-20, in which it was not retrieved on a rewarded run because the 20-pellet run ended the series. Thus, as the memory view predicts, 0 pellets was tracked more poorly in transfer in Group E-20 than in Group I-20. Thus, the findings for both Groups E-2 and I-2 and Groups E-20 and I-20 sug- 
gest a strong relationship between extinction and transfer in serial-learning tasks. ${ }^{1}$

\section{GENERAL DISCUSSION}

As indicated, the memory view of extinction is that the more strongly the memory of nonreward has been made to signal reward in acquisition, the more vigorously it will promote responding, and the more difficult it will be to convert it into a signal for nonreward in extinction. What is the case for the memory of nonreward, the present investigations suggest, is also the case for memories produced by small and large reward. In Experiments 1 and 2, they also promoted more vigorous responding, the more strongly they signaled reward, and in Experiment 3 they encountered greater difficulty in being converted from a signal for reward to a signal for nonreward in transfer, the more strongly they came to signal reward in original training. The present findings indicate, then, that serial learning and transfer in rats have much in common with other, better investigated forms of instrumental learning. This is a conclusion quite inconsistent with the rule-encoding view, strongly disconfirmed here, that serial-pattern learning in rats cannot be explained by available animal learning theories because it is a specialized, unique form of learning that requires human serial-pattern learning models for its explanation.

Having shown that the present findings confirm the memory view and disconfirm the rule-encoding view, the general conclusion suggested is that, on the basis of available evidence, the rule-encoding view is not acceptable-that, in effect, the findings of Hulse and Dorsky $(1977,1979)$ fail to offer an acceptable degree of support for the rule-encoding view. This is so, on the one hand, because the findings of Hulse and Dorsky do not exclude an alternative interpretation in terms of memory, and, on the other, because the experimental conditions employed by Hulse and Dorsky do not appear to differ substantially from those employed here when taken together with results reported by Capaldi and Molina (1979). Capaldi and Molina's results, like the present results, confirm the memory view and disconfirm the rule-encoding view. This combination of circumstances suggests that the results of Hulse and Dorsky are at least as likely to be due to memory as to rule encoding and that, at the least, those results cannot be seen as providing an acceptable degree of support for the rule-encoding view.

\section{Confounding of Memory Variables}

The two investigations reported by Hulse and Dorsky (1977) do not exclude a memory interpretation because, in them, memory variables of the sort shown to influence serial-pattern learning in the present investigations were confounded, and very extensively so. Consider first Experiment 2 of the Hulse and Dorsky (1977) report, in which the strongly monotonic 14-7-3-1-0 series led to better tracking of 0 pellets than did the weakly monotonic 14-5-5-1-0 series. In both series, $S^{1}$ signaled 0 pellets. However, the generalized reward-signal capacity received by $\mathrm{S}^{1}$ in each series is, because of the confounding of memory variables, completely indeterminate; so the possibility that $\mathrm{S}^{1}$ was supplied greater generalized reward-signal strength in the 14-5-5-1-0 series than in the 14-7-3-1-0 series cannot be excluded. Confounding becomes obvious once the rewarded memories in each series are identified. Such identification reveals not only that the two series contained different numbers of rewarded memories but also that they contained only one rewarded memory in common. Furthermore, the rewarded memories in the two series had different reward signal strengths, since they signaled different reward magnitudes. In the 14-7-3-1-0 series, there were three rewarded memories, $\mathrm{S}^{14}, \mathrm{~S}^{7}$, and $S^{3}$, which signaled 7,3 , and 1 pellet, respectively. However, in the 14-5-5-1-0 series, there were only two rewarded memories, $S^{14}$ and $S^{5}$, with $S^{14}$ signaling 5 pellets and $S^{5}$ signaling both 5 pellets and 1 pellet. On the basis of the findings obtained in this report, it is clear that, within a memory framework, the 14-7-3-1-0 series and the 14-5-5-1-0 series are not even remotely comparable. The same is true for the series examined by Hulse and Dorsky (1977) in their Experiment 1, the 14-7-3-1-0 series and the 14-1-3-7-0 series, in which different memories signaled 0 pellets, $S^{1}$ in the 14-7-3-1-0 series and $S^{7}$ in the 14-1-3-7-0 series.

\section{Memory and Transfer}

There were two major comparisons in the transfer phase of the Hulse and Dorsky (1979) investigation. In one of these, the monotonic one, it is clear that the memory view could not predict other than what was found by Hulse and Dorsky. In the other comparison, the nonmonotonic one, confounding of the memory variables was such that a memory interpretation cannot be excluded. In original training of the Hulse and Dorsky transfer investigation, 15 monotonic series and an even greater number of random series were employed. These series were two-, three-, and four-element patterns constructed from what was called an element set of $10,5,3,1$, or 0 pellets. For example, when the monotonic group, in which elements always decreased, received the four element pattern 10-5-3-1, the random group, in which elements could increase as well as decrease, received either the series 10-3-1-5 or the series 5-3-10-1. ${ }^{2}$ In transfer, 0 pellets was better tracked in a 16-9-3-1-0 monotonic series when previous training was monotonic and in a 16-1-3-9-0 nonmonotonic series when previous training was random.

In the 16-9-3-1-0 series, $\mathrm{S}^{1}$ signaled 0 pellets. Mem- 
ory variables were such that $S^{1}$ necessarily acquired a stronger tendency to signal reward when previous training was random. Thus, the memory view clearly predicts, as found by Hulse and Dorsky (1979), that better tracking of 0 pellets in the 16-9-3-1-0 series occurred when previous training was monotonic. Consider first those series in original training that contained 1 pellet. In monotonic series of this kind, 1 pellet ended the series (the 1-0 sequence was not allowed), and thus, in monotonic series, $\mathrm{S}^{1}$ could not directly acquire reward-signal capacity. But, in random series containing 1 pellet, reward could and did occur anywhere in the series, including prior to large-reward trials. Thus, in random series, $S^{1}$ could and did directly acquire a strong capacity to signal reward. Consider now series not containing 1 pellet in original training. In such monotonic series, as compared with random ones, the memories signaling reward were (1) less similar to $S^{1}$ and (2) signaled smaller reward. On both counts, then, $S^{1}$ received less generalized reward signal strength in monotonic series than in random ones. As an example, $\mathrm{S}^{1}$ obviously received less generalized reward-signal strength from $S^{10}$ in the 10-3 monotonic series than it did from $\mathrm{S}^{3}$ in the 3-10 random series, two series employed by Hulse and Dorsky (1979). There can be no doubt, then, that $S^{1}$ acquired greater reward-signal strength, both directly and indirectly, when previous training was random, and thus the transfer findings obtained by Hulse and Dorsky with the 16-9-3-1-0 series are completely consistent with the memory view.

Consider now the 16-1-3-9-0 series employed in transfer in which $S^{9}$ signaled 0 pellets. Due to confounding of memory variables, the generalized reward signal strength received by $\mathrm{S}^{9}$ in original training is indeterminate and thus a memory interpretation of the transfer findings obtained with the 16-1-39-0 series cannot be excluded. A good general example of such confounding may be provided by examining the 5-3 monotonic series and the 3-5 random series employed by Hulse and Dorsky in original training. In the monotonic series, $S^{5}$ signaled 3 pellets and in the random series $S^{3}$ signaled 5 pellets. Based on similarity, $S^{9}$ would receive more generalized reward-signal strength in the monotonic series, but, based on the magnitude of reward signaled, $\mathrm{S}^{9}$ would receive more generalized reward-signal strength in the random series. More extensive analysis is possible, but that would only confirm what was suggested above, that it cannot be determined whether $S^{9}$ received more generalized reward-signal strength in random or in monotonic series. Thus, a memory interpretation of the finding obtained with the 16-13-9-0 series cannot be excluded.

\section{Similarity of Experimental Conditions}

In two investigations, which, like the present ones, confirmed the memory view and disconfirmed the rule-encoding view, Capaldi and Molina (1979) found poorer tracking of 0 pellets under strongly monotonic series than under either mildly nonmonotonic series (Experiment 1) or completely nonmonotonic series (Experiment 2). Those investigations employed one trial each day, about a 4-min interval between runs, and series consisting of either four elements (Experiment 1) or three elements (Experiment 2). The findings of Capaldi and Molina, taken in combination with those reported here, appear to render unlikely the view that the memory view was confirmed and the rule-encoding view disconfirmed under experimental conditions that differ in important respects from those employed by Hulse and Dorsky (1977, 1979). The results of Capaldi and Molina indicated that tracking of 0 pellets is regulated by memory when the interval between runs is reasonably long ( $4 \mathrm{~min}$ ), and the present results indicate the same when the interval between runs was as short as that employed by Hulse and Dorsky, about $15-20 \mathrm{sec}$. While mainly two-element series were employed here, Capaldi and Molina employed three- and four-element series. In the original training of the Hulse and Dorsky (1979) investigation, in which rule encoding was assumed to occur, two- and three-element series were employed mainly with some four-element series, and while Hulse and Dorsky (1977) employed five-element series, such series are not very different from the fourelement series employed later by either Capaldi and Molina or Hulse and Dorsky. The maximum number of trials employed each day, one in Capaldi and Molina and two here, was well below the four each day employed by Hulse and Dorsky (1977) or the seven each day employed in original training by Hulse and Dorsky (1979). However, it is difficult to imagine why this variable would be important, why rats provided with more than two trials each day would stop employing memory and begin to encode rules. Accordingly, it seems quite unlikely that the experimental conditions employed in the investigations that have confirmed the memory view and disconfirmed the rule-encoding view differ substantially from those employed by Hulse and Dorsky $(1977,1979)$.

\section{REFERENCES}

CaPaldi, E. J. Effect of N-length, number of different N-lengths and number of reinforcements on resistance to extinction. Journal of Experimental Psychology, 1964, 63, 230-239.

Capaldi, E. J. Partial reinforcement: A hypothesis of sequential effects. Psychological Review, 1966, 73, 459-477.

Capaldi, E. J., Berg, R., \& Morris, M. D. Stimulus control of responding in the early trials of differential conditioning. Learning and Motivation, 1975, 6, 217-229.

Capaldi, E. J., Blitzer, R. A., \& Molina, P. Serial anticipation pattern learning in two-element and three-element series. Bulletin of the Psychonomic Society, 1979, 14, 22-24.

Capaldi, E. J., \& CAPAldi, E. D. Magnitude of partial reward, irregular schedules and a 24-hour ITI: A test of several hypotheses. Journal of Comparative and Physiological Psychology, $1970,72,203-209$. 
Capaldi, E. J., \& Molina, P. Element discriminability as a determinant of serial-pattern learning. Animal Learning \& Behavior, 1979, 7, 318-322.

Capaldi, E. J., \& Morris, M. D. The role of stimulus compounds in eliciting responses: Relatively spaced extinction following massed acquisition. Animal Learning \& Behavior, 1976, 4, 113-117.

Hulse, S. H. Cognitive structures and serial pattern learning by rats. In S. H. Hulse, H. Fowler, \& W. K. Honig (Eds.), Cognitive processes in animal behavior. Hillsdale, N.J: Erlbaum, 1978.

Hulse, S. H., \& Dorsky, N. P. Structural complexity as a determinant of serial pattern learning. Learning and Motivation, $1977,8,488-506$.

Hulse, S. H., \& Dorsky, N. P. Serial pattern learning by rats: Transfer of a formally defined stimulus relationship and the significance of nonreinforcement. Animal Learning \& Behavior, 1979, 7, 211-220.

LEONARD, D. W. Amount and sequence of reward in partial and continuous reinforcement. Journal of Comparative and Physiological Psychology, 1969, 67, 204-211.

\section{NOTES}

1. In interpreting the results of Experiment 3, directly acquired reward-signal strength was emphasized. An emphasis on generalized signal strength does not modify the conclusions reached. The reason is that generalized strength cannot exceed directly conditioned strength. Thus, $\mathrm{S}^{2}$ directly acquired greater signal capacity in Group I-20, in which it signaled 20 pellets, than in Group E-20, in which it signaled 2 pellets, and so $\mathrm{S}^{2}$ supplied $\mathrm{S}^{20}$ with greater generalized signal capacity in Group $1-20$ than in Group E-20. But $\mathrm{S}^{20}$ directly acquired greater signal capacity in Group E-20, in which it signaled 20 pellets, than in Group I-20, in which $\mathrm{S}^{20}$ did not directly signal any reward. It must be assumed that stronger reward-signal capacity will be possessed by $S^{20}$ when it signals 20 pellets (Group E-20) than when it signals 20 pellets only through generalization from $S^{2}$ (Group I-20); thus, as indicated above, 0 pellets was better tracked in transfer by Group I-20 than by Group E-20.

2. S. H. Hulse kindly provided us with a complete list of all reward series employed in original training of the Hulse and Dorsky (1979) investigation.
(Received for publication January 17, 1980; revisions accepted April 9, 1980 